\title{
Late onset pulmonary valvar stenosis after arterial switch operation for transposition of the great arteries
}

\author{
G Santoro, D Di Carlo, R Formigari, L Ballerini
}

Division of Pediatric Cardiology, Ospedale Bambino Gesù, P.zza S. Onofrio, 4 00165 - Rome, Italy G Santoro

R Formigari

L Ballerini

Division of Cardiac Surgery, Ospedale Bambino Gesù

D Di Carlo

Correspondence to: Dr Ballerini.

Accepted for publication 2 December 1997

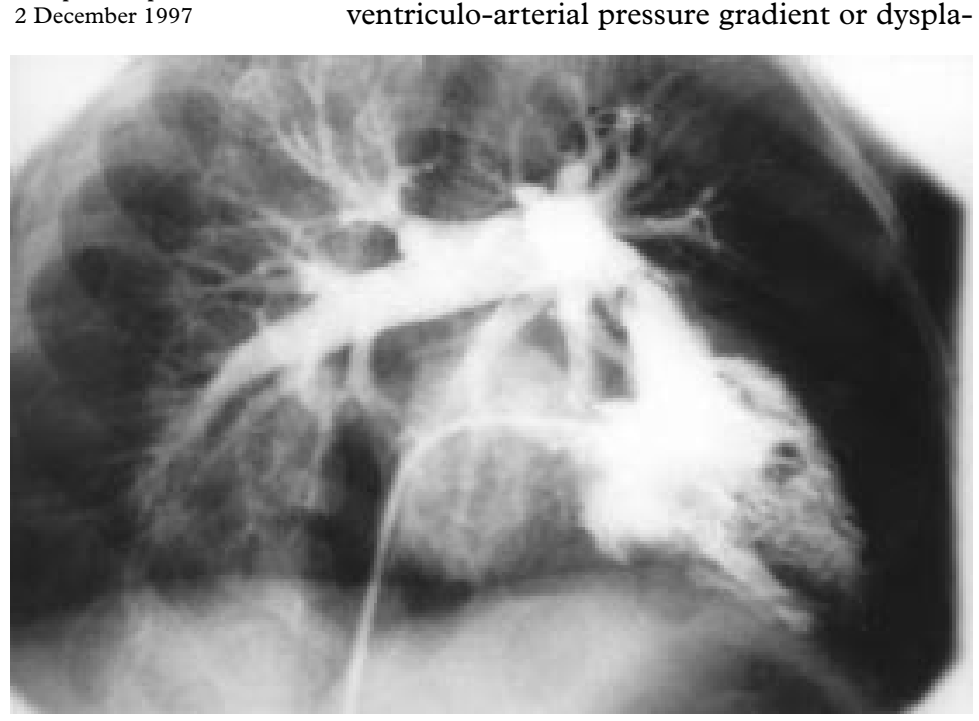

Figure 1 Right oblique view angiogramy showing the postprocedure pulmonary valve "doming" before the pulmonary balloon valvuloplasty. from late onset pulmonary valve stenosis (previously aortic valve) is exceedingly rare. ${ }^{6}$

We report two neonates who had the arterial switch procedure, in whom right ventricular outflow tract obstruction caused by neopulmoterm follow up. As with simple pulmonary valvar stenosis, balloon valvuloplasty proved to be effective in treating this rare late onset surgical complication.

\section{Case reports}

Two neonates, 1 and 5 days old respectively, were referred because of TGA-IVS. Significant
The arterial switch operation (Jatene procedure) is the treatment of choice for transposition of the great arteries with intact ventricular septum (TGA-IVS) in neonatal age, because of the low mortality and morbidity during long term follow up. ${ }^{1}$ Right ventricular outflow obstruction is the most frequent postoperative complication, ${ }^{2-4}$ occurring with sufficient severity to require reintervention in up to $10 \%$ of cases. ${ }^{15}$ Usually, the obstruction is either at the level of the pulmonary trunk or pulmonary bifurcation and, more uncommonly, in the right ventricular infundibulum. Conversely, right ventricular outflow obstruction resulting nary valve stenosis developed during medium ventriculo-arterial pressure gradient or dysplaflow obstruction is a common complication after surgery. The obstruction is usually located at the pulmonary trunk level, resulting from scarring of the anastomotic site or, more frequently, at the bifurcation of the pulmonary trunk owing to the traction on the pulmonary arteries after the Lecompte manoeuvre. ${ }^{14}$ Conversely, late onset stenosis of the neopulmonary valve (previously aortic valve) has been seldom reported. ${ }^{6}$ In our patients, the preoperative examination did not show native aortic valve dysplasia or stenosis, and direct inspection during surgery showed a trileaflet aortic valve. No significant right ventricular outflow obstruction early after surgery was recorded. Thus, the cause of the late onset neopulmonary valve stenosis is not well understood, although it could be supposed that, over time, a negligible, mild valvar dysplasia evolved into frank stenosis. It could also be speculated that the increased pulmonary trunk pressure secondary 
to the supravalvar stenosis may have caused turbulence and shear stress of the valve leaflets, resulting in progressive valvar stenosis. This form of right ventricular outflow tract obstruction was successfully treated by percutaneous balloon dilatation, with long lasting relief of the stenosis during medium term follow up, as previously reported for valvuloplasty performed in the first year of life. ${ }^{8}$ This favourable result of balloon angioplasty is at variance with previously reported experience. ${ }^{6}$

In conclusion, the pulmonary valve can be the site of right ventricular outflow tract obstruction after arterial switch operation and, unlike other types of right ventricular outflow obstruction secondary to arterial switch operation, ${ }^{6}$ it seems amenable to successful dilatation by percutaneous balloon valvuloplasty, thus avoiding further surgical procedures.
1 Kirklin JW, Barrat-Boyes BG. Complete transposition of the great arteries. In: Kirklin JW, Barratt-Boyes BG, eds. Cardiac surgery. Edinburgh: Churchill Livingstone, 1993:1383-467.

2 Wernowsky G, Hougen TJ, Walsh EP, et al. Mid-term results after arterial switch operation for transposition of the great arteries with intact ventricular septum: clinical, hemodynamic, echocardiographic and electrophysiologic data. Circulation 1988;77:1333-44.

3 Lupinetti FM, Bove EL, Minich LL, et al. Intermediateterm survival and functional results after arterial repair for transposition of the great arteries. F Thorac Cardiovasc Surg 1992;103:421-7.

4 Yacoub MH, Bernhard A, Radley-Smith R, et al. Supravalvular pulmonary stenosis after anatomic correction of transposition of the great arteries: causes and prevention. Circulation 1982;66:193-7.

5 Serraf A, Roux D, Lacour-Gayet F, et al. Reoperation after arterial switch operation for transposition of the great arteries. F Thorac Cardiovasc Surg 1995;110:892-9.

6 Nakanishi T, Matsumoto Y, Seguchi M, et al. Balloon angioplasty for postoperative pulmonary artery stenosis in 22:859-66.

7 Layman TE, Edwards JE. Anomalies of the cardiac valves associated with complete transposition of the great vessels. Am f Cardiol 1967;19:247-52.

8 Santoro G, Formigari R, Di Carlo D, et al. Mid-term Santoro G, Formigari R, Di Carlo D, et al. Mid-term
outcome after pulmonary balloon valvuloplasty in patients younger than 1 year of age. Am f Cardiol 1995;75:637-9. 